\title{
A New Principle of Motor Differential Protection based on Floating Threshold
}

\author{
Zhicheng Shen, Zhuoying Ke, Huiyuan Zhang \\ North China Electric Power University, Beijing, 102206, China
}

Keywords: motor protection, differential protection, floating threshold, the brake.

\begin{abstract}
The brake amount of traditional longitudinal differential protection of motor is a fixed value, so that for special extremes the relay is prone to maloperation or refuse to move. According to the motor fault characteristics, I take out a motor longitudinal differential protection principle which is proposed based on the amount of floating threshold brake. It will improve the reliability of the longitudinal differential protection, and the influence affected by transition resistance is reduced. What's more it can better remove the fault and prevent maloperation.
\end{abstract}

\section{Introduction}

Most of the motor in industrial applications is asynchronous motor. Motor differential protection is by comparing each phase of the flow of power on both ends of the traditional line, to judge whether it is the internal short-circuit fault. Similar as the longitudinal differential protection of other main electrical equipment, in order to ensure the sensitivity of the protection, motor microcomputer longitudinal differential protection also using the ratio brake characteristic.

The significance of large motor is more and more important for the power system, for example, the primary air fan of the single-auxiliary deploy affected the normal operation of the whole power plant, and the reliability-requirement of its protection is equivalent to generating set requirements. The original traditional longitudinal differential protection as affected by transition resistance is larger, and the non-fault phase current is affected by the fault phase will have a small fluctuations, making it difficult to meet the demand of the important motor relay protection reliability, sensitivity, selectivity and speed. To this end, this paper presents a longitudinal differential criterion motor protection based on floating threshold. And then, using the logical inference to diagnose and act based on the fault conditions.

\section{The traditional differential protection}

In the large capacity motor (above 2MW) which has six stator terminations, usually use longitudinal differential protection as the main protection of motor. At this time, the protection can provide higher sensitivity than other protections. The wiring principle (as shown in Figure 1) is that, two current transformers are compared (Transformer Models and ratios same) which at the current terminal and the neutral point, and the protection device action on the trip and signal according to the action current size.

The traditional differential protection is take advantage of the internal fault current difference in both sides of the fault to response the fault, and a greater impact by external system vibration.

The action amount is

The braking amount is

$$
\mathrm{I}_{\mathrm{op}}=\left|\mathrm{I}_{\varphi}+\mathrm{I}_{\varphi}{ }^{t}\right|
$$

$$
\mathrm{I}_{\mathrm{res}}=\frac{1}{2}\left|\mathrm{I}_{\Psi}-\mathrm{f}_{\Phi}\right|
$$

Among them, $\varphi=a, b, c$, and are the current of both sides of the motor.

The maximum unbalance current of longitudinal differential protection is according to avoid motor maximum starting current. 


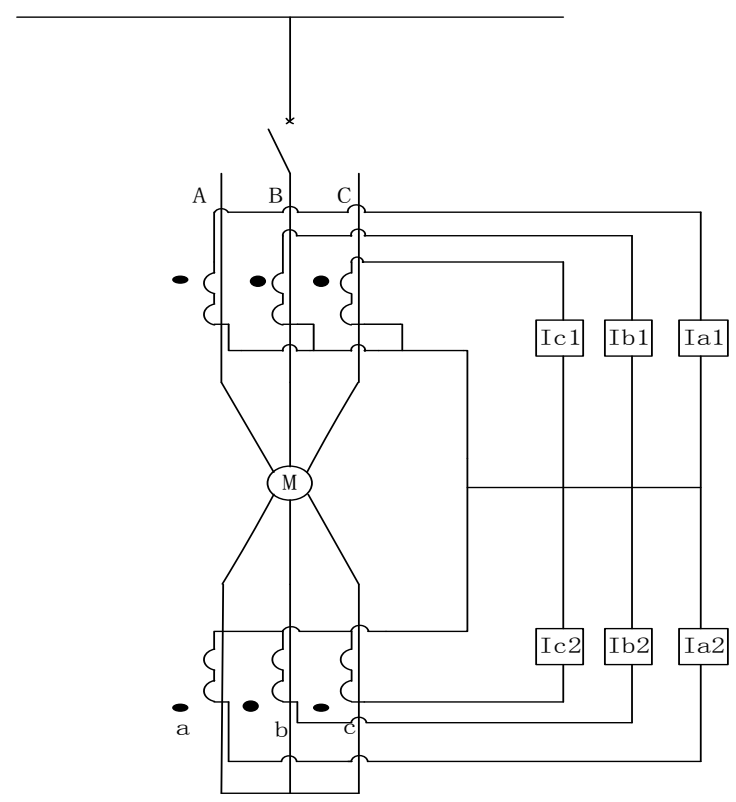

Fig.1: Motor differential protection wiring

\section{Put forward the floating threshold braking amount criterion}

When internal fault occur, fault phase transition resistance greatly make the action current become smaller; and the non-fault phase differential protection action quantity is not exactly zero, but is a small amount about the fault phase short circuit. In order to prevent maloperation and malfunction of relay protection, this paper proposes a new value about the restraint criterion, which is a logic inference using the original measurement (fixed threshold and floating threshold together constitute the braking amount).

Each phase momentum is

$$
\begin{gathered}
I_{\text {res }}^{a}=\operatorname{Kmax}\left\{I_{\mathrm{op}}^{b}, I_{\mathrm{op}}^{c}\right\}+I_{0} \\
I_{\text {res }}^{b}=\operatorname{Kmax}\left\{I_{\mathrm{op}}^{a}, I_{\mathrm{op}}^{c}\right\}+I_{0} \\
I_{\text {res }}^{c}=\operatorname{Kmax}\left\{I_{\mathrm{op}}^{b}, I_{\mathrm{op}}^{a}\right\}+I_{0}
\end{gathered}
$$

Then $\mathrm{I}_{\text {res }}^{\mathrm{a}}{ }^{x}$, $\mathrm{I}_{\text {res }}^{\mathrm{b}}{ }^{a}$ and $\mathrm{I}_{\text {res }}^{\mathrm{c}}{ }^{x}$ respectively are brake volume of a, b, c phase, $\mathrm{I}_{\text {op }}^{\mathrm{a}}, \mathrm{I}_{\mathrm{op}}^{\mathrm{b}}$ and $\mathrm{I}_{\mathrm{op}}^{\mathrm{c}}$ respectively are three phase action quantity of traditional criteria. $I_{0}$ Is fixed value, $\mathrm{K}$ as the braking coefficient.

Thus, you can get a new principle motor differential protection based on floating threshold based on the above formula. The expression is

$$
\begin{gathered}
I_{\mathrm{op}}^{a}>I_{\text {res }}^{a}=\operatorname{Kmax}\left\{I_{\mathrm{opp}}^{b}, I_{\mathrm{op}}^{c}\right\}+I_{0} \\
I_{\mathrm{op}}^{b}>I_{\text {res }}^{b}=\operatorname{Kmax}\left\{I_{\mathrm{op}}^{a}, I_{\mathrm{op}}^{c}\right\}+I_{0} \\
I_{\mathrm{op}}^{c}>I_{\text {res }}^{c}=\operatorname{Kmax}\left\{I_{\mathrm{op}}^{b}, I_{\mathrm{op}}^{a}\right\}+I_{0}
\end{gathered}
$$

If the measured current meet the above expression, relay protection will act and fault clearing. $I_{0}$ Bases on the maximum unbalance current, that generally affected by the motor starting current and different transformer models, time difference and other issues. When fault happen in the protection zone and short circuit current is large, floating criterion will dominate in the braking amount, we need to choose reasonable $\mathrm{K}$ value to effectively prevent the non-selective action of the protection. 


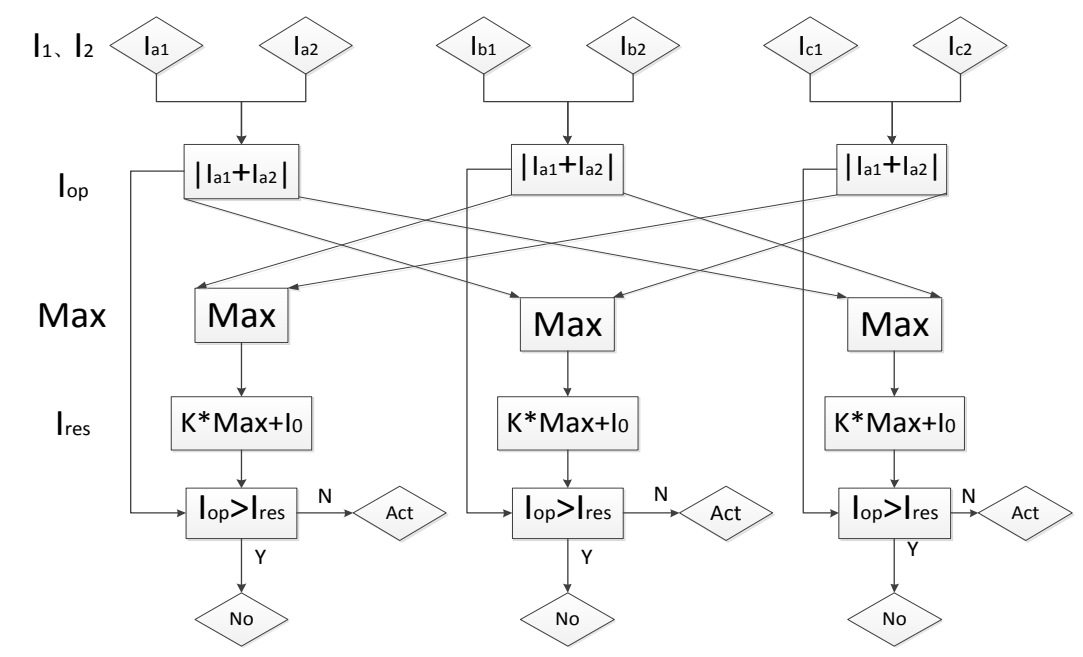

Fig.2: Logic diagram

\section{The simulation and verification}

I will use PSCAD/EMTDC software to realize the function shown in figure 1 and figure 2, and using the primary air fan parameters of Datang Wuan power plant as the parameters of the motor. And the simulation will run in different conditions, such as internal faults and external faults, and the single phase fault, two-phase fault, two-phase-ground fault, and three-phase faults in different fault locations, to verify the sensitivity and reliability of this criterion.

In the simulation, $\mathrm{K}$ is 0.1 , the $I_{0}=0.2 \mathrm{p}$. u., and the base current value is $251 \mathrm{~A}$.

The figure 3 is the action current and braking current of each phase in the normal operation below ,so that, the amount of braking much more than the amount of action, and the protection does not act reliable to ensure the normal operation of the system.

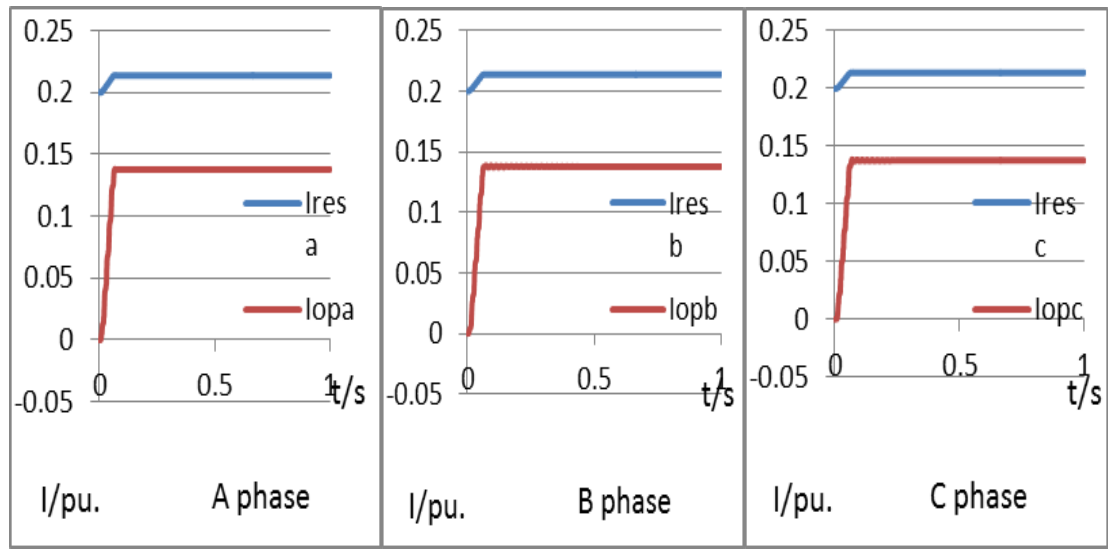

Fig.3: The action current and braking current amplitude of each phase in normal

If there is an inner fault happens, the current on both sides of the fault phase were unbalance and non-fault phases are less affected. At this time, the action current of the fault phase increases and the braking current less affected. Braking current in non-fault phase increased with the action current in fault phase, and current action basically unchanged. At this point, the fault phase act reliable and non-fault phase no action to realize the selectivity of relay protection. As shown in Fig.4, these are the baking currents and action currents of each phase when single phase ground fault happens. And Fig. 5 is the three-phase fault results. 


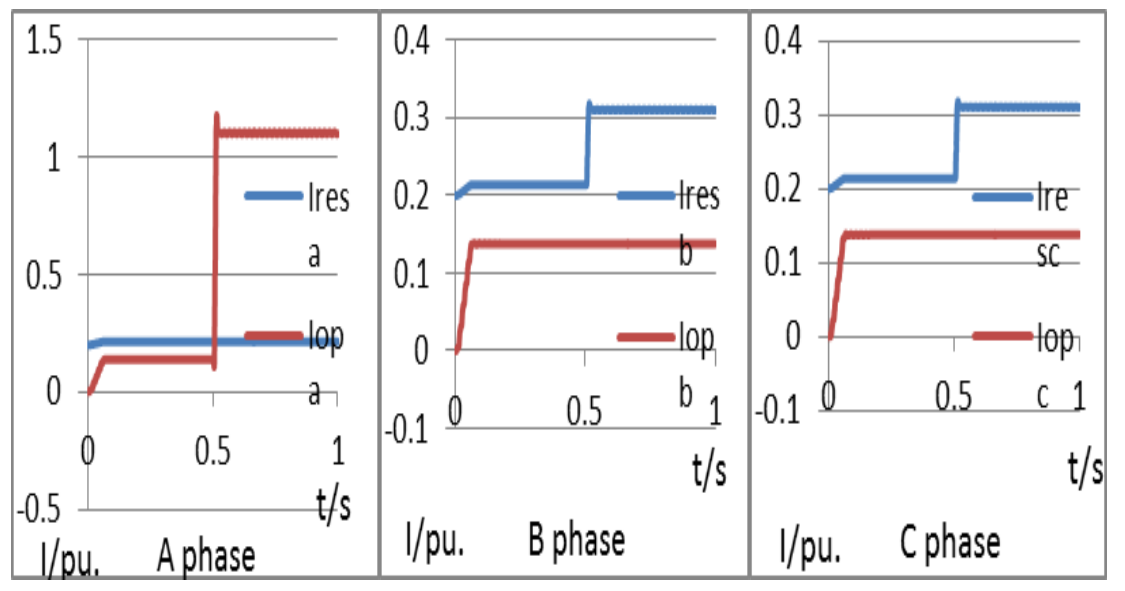

Fig.4: The action current and braking current amplitude of each phase when a phase ground fault happens inner area

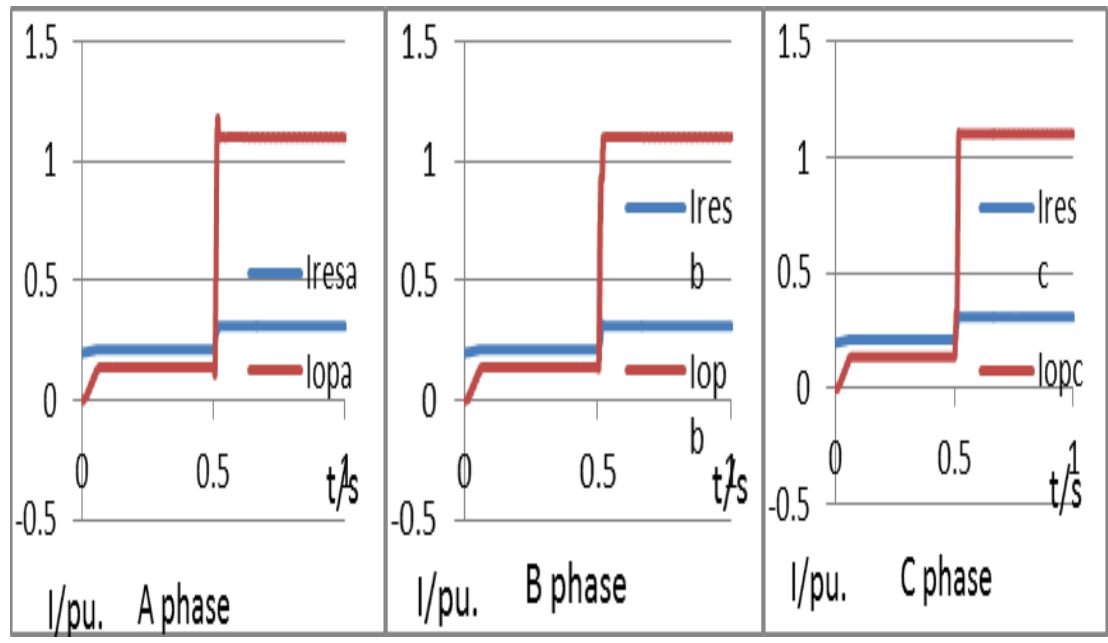

Fig.5: The action current and braking current amplitude of each phase when three-phase fault happens inner area

When a fault protection outside the region, action current of non-fault phase basically no change, and the change of braking amount that affected by the action current of fault phase is also small. But because of the existence of the I_0, the braking amount is much bigger than action amount and no false action. The action current of the fault phase are reduced, and the amount of braking is mainly unchanged which controlled by action current of non-fault phase. Thus, it is reliable no-act. Simulation results are shown as the Fig.6 is unbalance fault and Fig.7 three-phase fault.

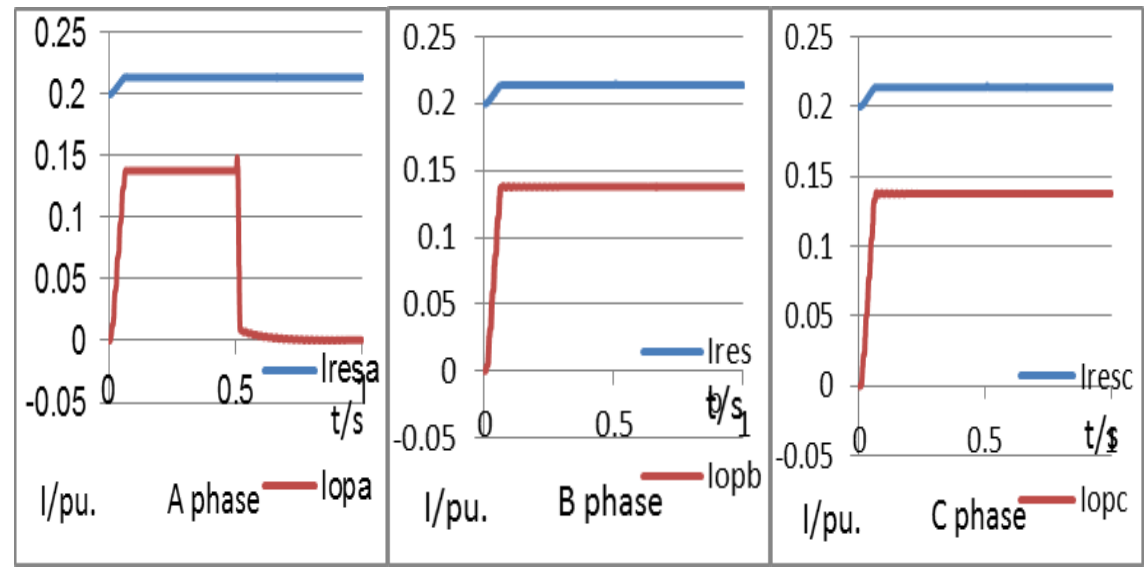

Fig.6: The action current and braking current amplitude of each phase when A-phase-ground fault happens outer area 


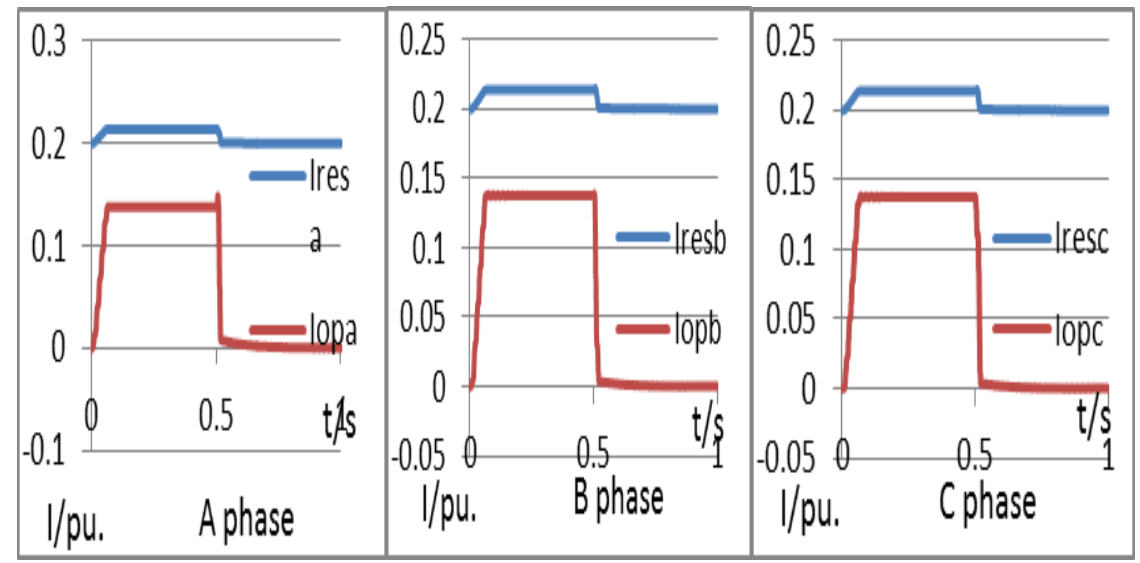

Fig.7: The action current and braking current amplitude of each phase when three-phase fault happens outer area

From the above simulation results, when internal fault occurs in motor protection scope, the protection criterion can operate correctly and no maloperation and false action. When fault occurs out of the motor protection scope, the protection criterion can brake correctly and no false action, meet the require about the reliability, sensitivity, selectivity of system operation.

When the single-phase grounding fault happens via a $10 \Omega$ resistor within the protection zone of the motor, current action and braking current as shown below (Fig. 8). Obviously, the criterion can still act correct.

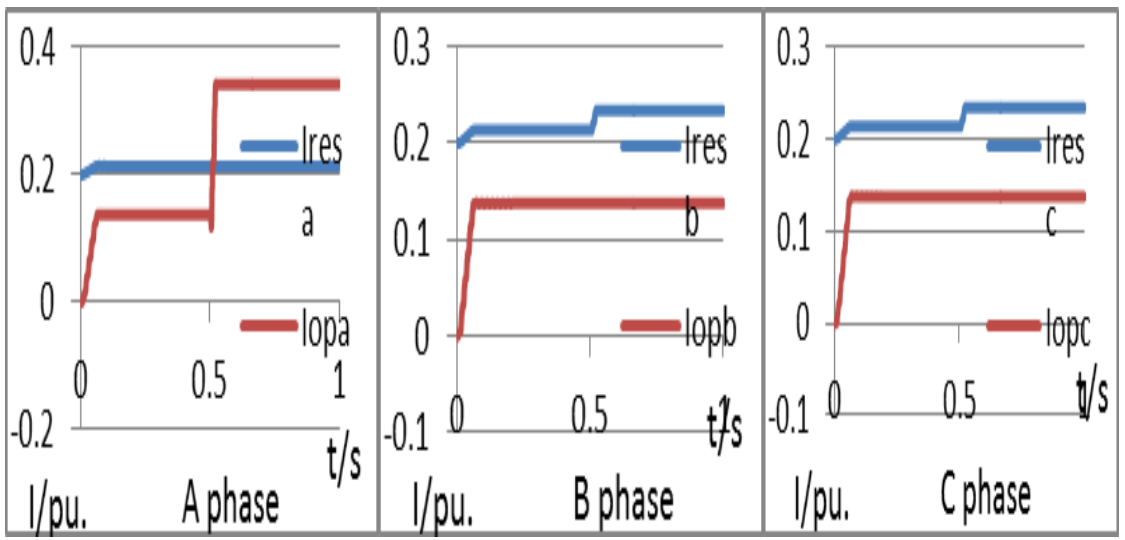

Fig.8: The action current and braking current amplitude of each phase when a phase ground fault happens via a $10 \Omega$ resistor inner area

The simulation results show that if asymmetric fault occurs in motor protection scope, because of the floating threshold effect, this criterion makes the braking current of non-fault phase becomes large and action current constant. So it could prevent the maloperation of the protection. If threephase fault occurs in the motor protection scope, the action current of fault phase is very big due to the effect of the fault current, and the braking current size limit by K. So it could act correctly. When there is a fault happens out of the protection, due to a fixed threshold $\left(I_{0}\right)$ effects, the brake amount was far more than that amount of action. So it could prevent the maloperation of the protection. For the ground fault through impedance, this criterion can also give a correct action.

\section{Conclusions}

This paper studies the motor differential protection whose baking amount based on the floating threshold and the fixed threshold, and the theoretical analysis and simulation results show that:

This relay protection method can eliminate the influence of transition resistance on motor differential protection;

This criterion could be able to correctly distinguish the internal faults and external faults, and distinguish fault phase or non-fault phase. 


\section{References}

[1] He Jia Li, Li Yongli, Dong Xinzhou, Li Bin. The theory of power system protection [M]. China Electric Power Press, 2010

[2] Li Guangqi. Power system transient analysis [M]. China Electric Power Press, 2007

[3] Yuan Weifang. 3800kW high voltage motor relay protection configuration [J]. Metallurgical electric, 2013

[4] Jiang Tong, Lv Yanping .Bergeron model of the longitudinal current protection criterion based on the new [J]. Electric automation, 2014, 36 (3)

[5] Guo syndrome. UHV long segregated phase current differential protection principle of new [D]. Tianjin: Doctoral Dissertation of Tianjin University, 2004

[6] Li Bin, Fan Ruiqing, He Jiali. Motor magnetic balanced differential protection setting calculation [J]. Power system protection and control, 2010.38 (13). 